\title{
Chaos in models of the solar neighbourhood
}

\author{
D. Chakrabarty ${ }^{1}$ and I. V. Sideris ${ }^{2}$ \\ 1 School of Physics \& Astronomy, University of Nottingham, Nottingham NG7 2RD, UK \\ e-mail: dalia.chakrabarty@nottingham.ac.uk \\ 2 Institute for Theoretical Physics, University of Zürich, Winterthurerstrasse 190, Zürich 8057, Switzerland \\ e-mail: sideris@physik.unizh.ch
}

Received 21 December 2007 / Accepted 18 April 2008

\section{ABSTRACT}

\begin{abstract}
Aims. We quantify the amount of chaos that exists in the local phase space.
Methods. A sample of orbits from four different models of the solar neighbourhood phase space are analysed by a new chaos identification (and quantification) technique. While three of the models we used bear the signature of the perturbation due to both the Galactic bar and the spiral pattern, the last of the models is a bar-only one. We explore the models by comparing the corresponding values of chaos strength that are induced at the various energy levels.

Results. We find that all the viable models that have been demonstrated to successfully reproduce the local phase space structure models that include the bar as well as the spiral - bear strong chaoticity, though the model that implies the highest degree of chaos is the one in which overlap of the major resonances of the bar and the spiral occurs. The bar-only model is found to display regularity. We advance chaos as primarily responsible for the splitting of the Hyades-Pleiades mode (the larger mode) of the local velocity distribution.
\end{abstract}

Key words. chaos - Galaxy: solar neighbourhood

\section{Introduction}

The availability of transverse velocities of nearby stars from Hipparcos has facilitated the construction of the local phase space distribution (Fux 2001; Skuljan et al. 1999; Dehnen 1998). In contradiction to the conventional idea of stellar dynamics, all representations of this distribution manifest strong non-linearity and multi-modalness. This clumpy nature of the solar neighbourhood velocity distribution $(f)$ has been addressed in Fux (2001); Quillen (2003); Dehnen (2000); Chakrabarty (2007); Famaey et al. (2005); de Simone et al. (2004) and others; consensus appears to be emerging as to the origin of the basic bimodal nature of the distribution in terms of scattering off the outer Lindblad resonance of the Galactic bar $\left(O L R_{\mathrm{b}}\right)$.

However, a dynamical basis for the existence of the other structure (such as the Hyades, Pleiades, Sirius, Coma Berenicus stellar streams) has not been explored sufficiently Chakrabarty (2007, hereafter, Paper I) concluded that the observed phase space structure is due to the dynamical influence of the Galactic bar and 4-armed spiral pattern; the influence of the bar alone or the spiral alone was reported to be insufficient in explaining the present day observations of the solar neighbourhood Quillen (2003). invoked the chaos caused by the overlapping of the $O L R_{\mathrm{b}}$ and the 4:1 resonance of the Galactic spiral pattern to explain the chaos-dominated state of the local disc, which, Quillen (2003) suggested, is responsible for the clumpy nature of $f$.

In spite of these investigations, the quantification of the degree of chaos in the solar vicinity has not been undertaken yet. This is of interest in interpreting the state of the local patch in the disc and extrapolating this notion to the understanding of the Galactic disc as a whole, as well as of outer discs in external spiral systems. The former of these motivations is set to get a boost in the near future, with the quantity and spatial cover promised in the data from the upcoming GAIA mission. Here we present a new technique for estimating the amount of chaos that is induced in the solar neighbourhood by the Galactic bar and spiral pattern. The different models used in Paper I will be analysed by the technique advanced in Sideris (2006). Thus, the aim of this paper is to evaluate the extent of chaos in the solar neighbourhood and examine the possible connection between the identified chaos and the local phase space structure.

This paper is organised as follows. The following section deals with the models, while in Sect. 3 the equations of motion are briefly discussed. The chaos quantification technique is advanced in Sect. 4 and the recovered results are presented herein. Section 5 is dedicated to a discussion of some aspects of the work. The paper is rounded off with the concluding remarks in Sect. 6.

\section{Models of the local disc}

As said before, we endeavour to infer the degree of chaos present in the vicinity of the Sun by gauging the chaoticity of the solar neighbourhood models that were presented in Paper I. Thus, the justification of the choice of the relevant parameters will not be repeated here; rather, it is the quantification of the inherent chaos in each of these models that is discussed below.

In Paper I, an annulus in the outer part of the Galactic disc was modelled by test particle simulations, in which a warm exponential disc was stirred by the bar or a spiral pattern alone, or by both these perturbations jointly. In these simulations, the Galactic disc is assumed to be ideal with the disc stars assumed to be drawn from a 4D phase space. A sample of phase space coordinates were chosen from a model initial phase space distribution function (chosen to ensure an exponential surface mass density profile and enough warmth to attain the velocity dispersions 
and vertex deviation observed in the solar neighbourhood today). These coordinates were allowed to evolve with time in the presence of the potential of the disc and the perturbation(s), i.e. the bar or (and) spiral. The bar was modelled as a rigidly rotating quadrupole (see Eq. (1) in Paper I) with a perturbation strength that is half the strength of the bar used in Fux (2001). The spiral pattern is modelled as a logarithmic spiral that is 4-armed (Vallée 2002) and tightly wound (pitch angle of $15^{\circ}$ ), as the model spiral pattern used by Johnston et al. (2001); this choice of number of arms and a low pitch angle also ties in with the suggestion of Melnik (2006); Bissantz et al. (2003); Englmaier \& Gerhard (1999); Vallée (2002). The initial disc configuration is characterised by a logarithmic potential to ensure flat rotation curve and a doubly-cut out distribution function (Evans \& Read 1998) that ensures an exponential surface stellar mass density profile. This distribution function is parametrised by a hotness parameter that is maintained high enough to ensure the recovery of velocity dispersions and vertex deviation that match with the observed values of these quantities in the solar neighbourhood today.

The orbits were recorded in the annulus between $R=1.7 R_{\mathrm{CR}}$ to $R=2.3 R_{\mathrm{CR}}$, where $R_{\mathrm{CR}}$ is the corotation radius of the bar; $O L R_{\mathrm{b}}$ occurs at $1.7 R_{\mathrm{CR}}$ for the above-mentioned choice of the disc potential. In this work, all lengths are expressed in units of $R_{\mathrm{CR}}$ and given the scale-free nature of our disc configuration, the physical value of $R_{\mathrm{CR}}$ is not relevant. An important parameter that was varied to define the individual models is the ratio between the pattern speeds of the bar $\left(\Omega_{b}\right)$ and the 4-armed spiral $\left(\Omega_{\mathrm{s}}\right)$. In every other respect, the bar+spiral models are similar to each other. The bar-only model is similar to the bar+spiral models in every respect except that there is no perturbation from the spiral in this model. The spiral-only model on the other hand, excludes the effect of the bar. Thus, the 5 models used in Paper I are:

- bar alone perturbing the disc;

- bar and spiral acting in concert with $\Omega_{\mathrm{b}} / \Omega_{\mathrm{s}}=55 / 25$;

- bar and spiral acting in concert with $\Omega_{\mathrm{b}} / \Omega_{\mathrm{s}}=55 / 21$;

- bar and spiral acting in concert with $\Omega_{\mathrm{b}} / \Omega_{\mathrm{s}}=55 / 18$;

- spiral acting alone.

From Paper I we learn that out of these 5 models, the first four were found to give rise to a phase space structure that is reminiscent of the observed structure (checked via a hypothesis testing technique), though the bar-only model was rejected on further dynamical grounds. In particular, the bar+spiral model that is characterised by $\Omega_{\mathrm{b}} / \Omega_{\mathrm{s}}=55 / 21$ is the one that ensures that the inner Lindblad resonance of the spiral $\left(I L R_{\mathrm{S}}\right)$ occurs at the same physical location as the $O L R_{\mathrm{b}}$. Thus, this is the model that corresponds to overlap of the major resonances of the two perturbations and therefore augers interesting dynamical consequences.

\section{Equations of motion}

In this section, the stellar equations of motion are discussed. Below is presented the Hamiltonian in an intertial frame, in galactocentric coordinates $x_{i}$, for $i=1,2$ and their conjugate momentum (or velocity $v_{i}$ ), given the logarithmic potential of the background disc $\left(\sim-\ln (R)\right.$, where $\left.R=\sqrt{x_{1}^{2}+x_{2}^{2}}\right)$ and the perturbations due to the quadrupolar bar $\left(\Phi_{\text {bar }}\right)$ and the logarithmic $m=4$ spiral pattern $\left(\Phi_{\text {spiral }}\right)$.

$\mathcal{H}=\sum_{1}^{2} v_{i}^{2}+\ln (R)+\Phi_{\text {bar }}+\Phi_{\text {spiral }}$, where the potential of the bar and the spiral in our scale-free units (i.e., all lengths are expressed in units of the bar co-rotation radius), in the inertial frame, at time $t$ are

$$
\begin{aligned}
\Phi_{\mathrm{bar}} & =-\epsilon_{\mathrm{bar}} \frac{\cos 2\left(\phi-\Omega_{b} t\right)}{R^{3}} \\
\Phi_{\text {spiral }} & =-\epsilon_{\text {spiral }} K(\alpha, m) e^{i\left[m\left(\phi-\Omega_{s} t\right)\right]} R^{i \alpha-\frac{1}{2}}
\end{aligned}
$$

Here $\alpha=m \cot (i)$, where $i$ is the pitch angle of the spiral and $m$ is the number of arms in the pattern $\left(i=15^{\circ}\right.$ and $m=4$ for our models). $K(\alpha, m)$ is the Kalnajs gravity function as defined in Eq. (13) of Chakrabarty (2004). Also, $\epsilon_{\text {bar }}$ and $\epsilon_{\text {spiral }}$ are the bar and spiral strengths, defined in terms of the fractional contribution of the particular perturbation to the field due to the background disc $(\approx 3.6 \%$ for the bar and the spiral). Lastly, here $\phi$ is the azimuthal coordinate: $\phi=\tan ^{-1}\left(x_{2} / x_{1}\right)$. See Sect. 2.2 of Chakrabarty (2004) for a detailed discussion of the equations of motion.

Thus, in the inertial frame, the equations of motion are

$\ddot{\mathbf{x}}_{\mathbf{i}}=\frac{-\mathbf{x}_{\mathbf{i}}}{R^{2}}-\nabla \Phi_{\text {bar }}-\nabla \Phi_{\text {spiral }}$

When the only imposed perturbation is the bar, recording the orbits in the frame of the bar implies that the Jacobi integral is:

$\mathcal{H}_{\mathcal{J}}=\sum_{1}^{2} v_{i}^{2}+\ln (R)-\epsilon_{\mathrm{bar}} \frac{\cos 2 \phi}{R^{3}}$

Thus, in this case, $\mathcal{H}_{\mathcal{J}}$ is an integral of motion and the surfaces of section that are recovered for this 4D phase space, by setting $v_{y}=0$, is $2 \mathrm{D}$.

However, in the multiple pattern speed scenario, the Hamiltonian is no longer the Jacobi integral; thus, when the spiral pattern is included as the second perturbation, and the orbits recorded in the frame rotating with the bar, the orbital energy is

$$
\begin{aligned}
\mathcal{H}_{\mathcal{J}}= & \sum_{1}^{2} v_{i}^{2}+\ln (R)-\epsilon_{\mathrm{bar}} \frac{\cos 2(\phi)}{R^{3}} \\
& -\epsilon_{\text {spiral }} K(\alpha, m) \mathrm{e}^{\mathrm{i}\left[m\left(\phi-\left(\Omega_{s}-\Omega_{b}\right) t\right)\right]} R^{i \alpha-\frac{1}{2}} .
\end{aligned}
$$

It is obvious that the quantity $\mathcal{H}_{\mathcal{J}}$ in Eq. (5) returns to the same value periodically for period $T=m * \pi /\left(\Omega_{\mathrm{b}}-\Omega_{\mathrm{s}}\right)$, so if data are recorded stroboscopically every such period, $\mathcal{H}_{\mathcal{J}}$ is equivalent to an integral of motion. Then out of the recorded points per orbit (which are recorded only when $t=T$ ) one can construct two dimensional surfaces of section by employing a second constraint, in our case by choosing to plot only the points which have $v_{y}=0$. Any other constraint one may impose, e.g. $v_{x}=0$, should give the same results regarding the percentages of chaotic and regular orbits or strengths of chaos, since we quantify the same set of orbits but at a different surface section.

\section{Quantification of chaos and results}

The quantification of chaos for the orbits of this paper was achieved by the use of a new measure, first introduced by Sideris (2006). This technique is based on the recognition of smooth patterns in the signals associated with an orbit. It was shown in the original paper that the extrema of regular orbits correlate in such ways so to build smooth curves. This inherent smoothness, typically hidden inside the signal, can then be implemented to define a measure of regularity, through an intricate interpolation 


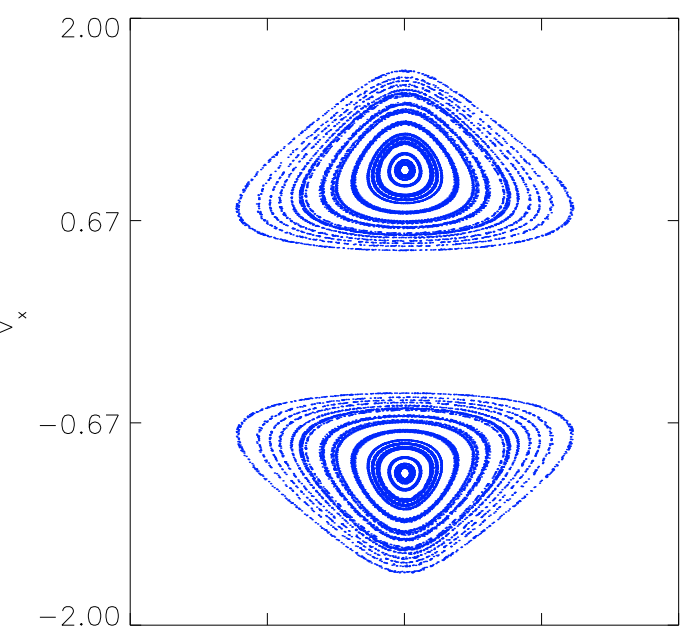

Fig. 1. Poincaré section for the bar-only model, at the energy of -0.75 . The white inner regions mark the part of $x-v_{x}$ space that is not populated by orbits for the specific implementation of our numerical experiment. The blue lines are invariant curves (i.e. curves representing the $4 \mathrm{D}$ regular orbits in the 2D Poincaré space).

scheme. The simple picture is that the smoother the curves, the more regular the signal.

A chaotic orbit usually evolves in a divided phase space - a phase space which is characterised by both regular and chaotic regions (Contopoulos 2002; Sideris 2008). In such a regime, any chaotic orbit (provided it is integrated for long enough timescales) will experience two kinds of dynamical epochs: strong or wild chaos and weak or sticky chaos (Shirts \& Reinhardt 1982; Contopoulos 2002). Strong chaos is associated with motion of the orbit far away from the regular islands. Such motion is completely unpredictable, and the chaotic orbit attempts to cover broad parts of the chaotic sea that is energetically available to it. When the orbit moves close to the regular islands it becomes trapped for a long time around them; in practice, it attempts to mimic regularity. The closer to a regular island the chaotic orbit moves the more persuasive is this mimicry.

The pattern method can identify when an orbit gets into weakly chaotic regimes. Semi-smooth curves correlating extrema of the signal of the orbit appear in that epoch of its evolution. The big advantage of the pattern method is that it treats orbits as sets of segments, piece by piece, and not as one monolithic entity as other measures typically do. This is how it achieves to distinguish parts of the orbit where weak chaos is experienced.

We applied this method to the orbits associated with the aforementioned simulations. For every simulation, a number of orbits corresponding to a given value of energy, were randomly extracted. This exercise was repeated in several different energy bands and the chaos quantification followed.

For the bar only model for the six energies evolved (from -0.3 to -1.5 ) no chaos was found. In Fig. 1, we show a surface of section that is constructed for orbits characterised by an energy of -0.75 . All the surfaces of sections presented herein are recorded for the orbits crossing the plane $v_{y}=0$.

The results for six different energies for the ratio $21 / 55$ can be seen in Fig. 2. Similar pictures hold true for models 18/55 and $25 / 55$. In all three models it is obvious that chaos is very strong for high energies but reduces as energy decreases.
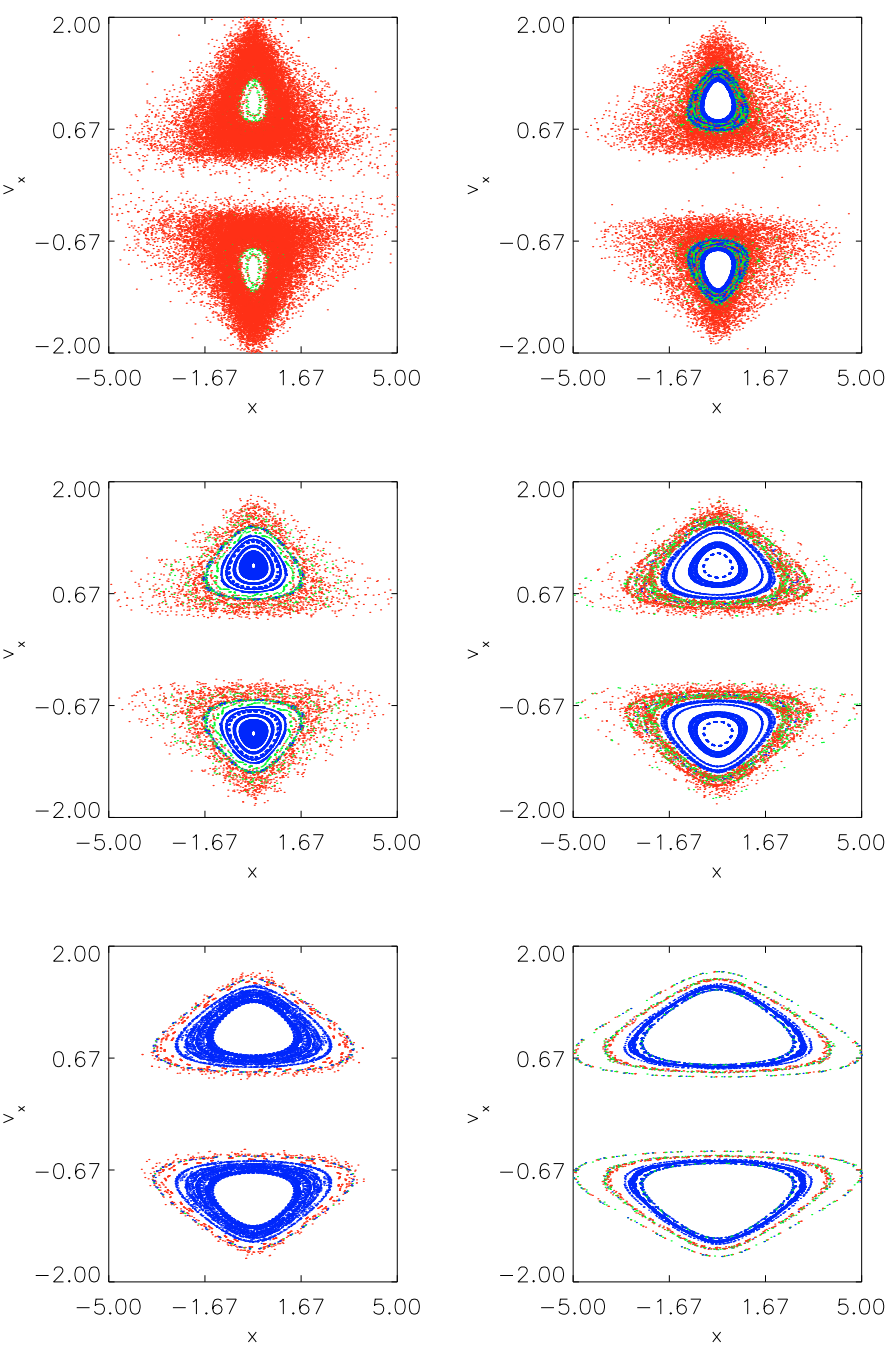

Fig. 2. Surface of sections of orbits integrated in the model with a spiral to bar pattern speed ratio of 21/55. Red signifies strong chaos, green signifies weak chaos, and blue signifies regularity. Each panel represents a surface of section plot for a particular energy value; top left panel corresponds to $J=-0.300$, top right to -0.5 , middle left to -0.75 , middle right to -1.0 , bottom left to -1.25 , and bottom right to orbits corresponding to energy of -1.5 . It is evident that chaos decreases as energy decreases.

To compare the chaos inducing ability of the different models, the fraction of the regular and (strongly and weakly) chaotic orbits is shown in Fig. 3. These plots show the percentage of chaotic orbits appearing in the three models. One may notice that the case $21 / 55$ is quantified as more chaotic than the other cases.

In Fig. 4 the chaos strength is plotted with respect to the energy for the four models $\Omega_{\mathrm{s}} / \Omega_{\mathrm{b}}=18 / 55,21 / 55,25 / 55$ and the bar-only.

\section{Discussions}

Our chaos quantification technique helps shed light on the models. We find that at the higher energies, the model that manifests the highest chaos is the model that ensures resonance overlap (the 21/55 model). This is in line with our expectations of course, but it is also interesting to note that the chaos induced by the other bar+spiral models is not much less either. At the same time, from Paper I, we know that all three of the bar+spiral 


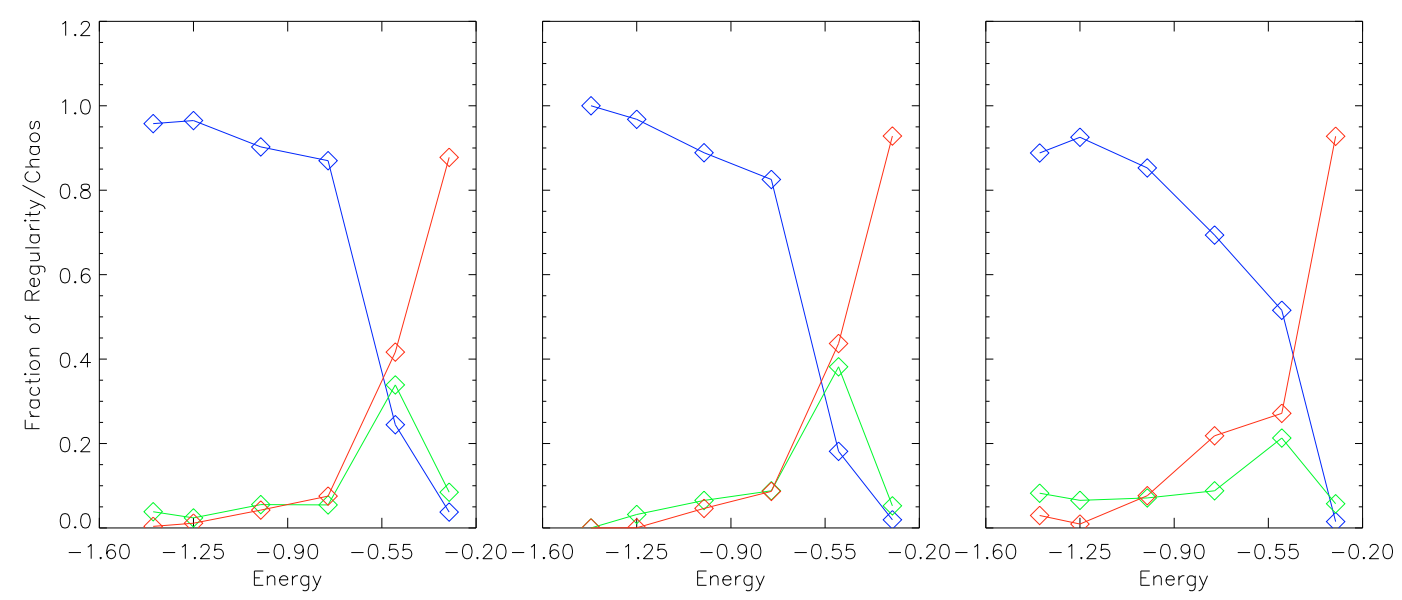

Fig. 3. Fractions of chaotic orbits (in red), weakly chaotic orbits (in green) and regular orbits (in blue), plotted as functions of energy, for the three bar+spiral models 18/55 (left), 21/55 (middle), 25/55 (right).

models were successful in explaining the observed structure of the local phase space. This adds weight to the suggestion that chaos is responsible for the clumps of the local velocity space. (Of course, this is only part of the story, since scattering off the $O L R_{\mathrm{b}}$ and the effects of minor resonances of the bar and the spiral are also important, as reported in Paper I).

To understand the trends in our results, we need to invoke the following: $I L R_{\mathrm{s}}$ is an "angular momentum emmitter" (LyndenBell \& Kalnajs 1972), the basic effect of which is to "stir without heating" (Sellwood \& Binney 2002). This idea that the $I L R_{\mathrm{S}}$ is the location from which stars are driven outwards, is corroborated by the experiments of Chakrabarty (2004). Now in our modelling, we choose to record our orbits in an annulus that extends from $R=1.7 R_{\mathrm{CR}}$ to $2.3 R_{\mathrm{CR}}$, where $R_{\mathrm{CR}}$ is the corotation radius of the bar. Thus, the occurrence of $I L R_{\mathrm{S}}$ at $R<1.7 R_{\mathrm{CR}}$ (the $25 / 55$ model) implies that stars will be pushed into the relevant annulus from lower radii than when $I L R_{\mathrm{S}}$ concurs with the physical location of $O L R_{\mathrm{b}}$. In the case $I L R_{\mathrm{S}}$ occurs at $1.7 R_{\mathrm{CR}}<R<2.3 R_{\mathrm{CR}}$, (the $18 / 55$ model), a part of the annulus will be depleted at the cost of the parts at radii around $2.3 R_{\mathrm{CR}}$. Thus, for the 25/55 model, more stars will be entering our annulus from lower energies than in the other two models. Now, in a smooth, unperturbed background potential, stars at lower radii are also more energetic than those at higher radii. This implies that in the absence of resonances due to imposed perturbations, there would have been more high-energy stars recorded for the $25 / 55$ case than in the $18 / 55$ or $21 / 55$ models.

This situation is of course challenged once the perturbations are introduced - in particular, proximity to resonance overlap indicates enhanced chaoticity in the recorded orbits. The relative excess in the energy of the recorded orbits, as implied by the $25 / 55$ model is surpassed, more at higher energies than lower, by the strength of chaos that is a signature of the resonance overlap case. This explains the relative trends in chaos strength that is noticed in the different models (Fig. 4).

We conclude that the observed phase space structure in the solar neighbourhood (particularly the splitting of the Hyades-Pleiades mode) is, to a large extent, chaos induced. But this chaos does not necessarily have to be triggered by resonance overlap (in contradiction to what Quillen 2003 suggested). In fact, the presence of chaos is found to be actuated by the spiral potential. We say this since our results indicate that the bar potential alone is insufficient in producing chaos. This contradicts the suggestion by Fux (2001). The bar that was used in

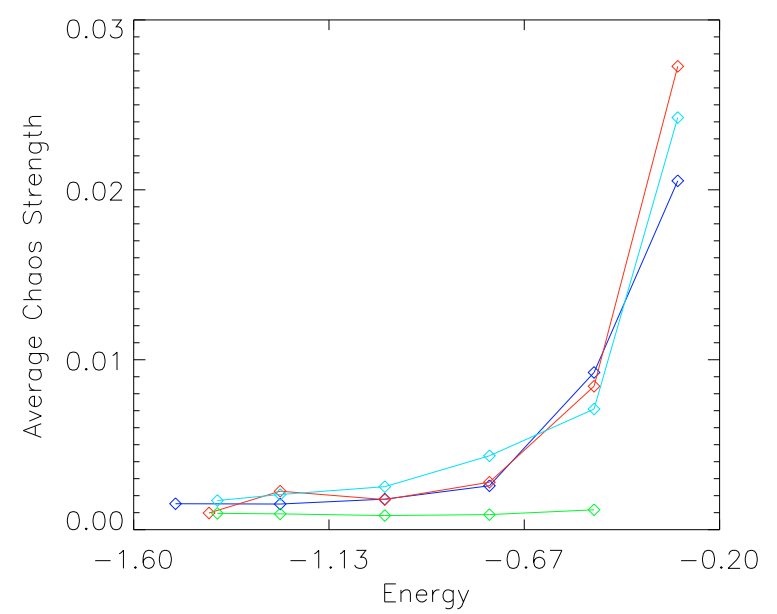

Fig. 4. Average strength of chaos against energy, for the four models $18 / 55,21 / 55,25 / 55$ and bar only. Blue signifies the 18/55 model, red $21 / 55$, cyan $25 / 55$ and green the bar only model.

the modelling in Paper I (our models) imposes a field of $3.6 \%$ of that of the background disc, nearly half of what was used by Fux (2001). Thus, it may be argued that it is this low a bar strength that was incapable of heating the disc enough; after all, as shown in Chakrabarty (2004), disc heating increases rapidly with increases in bar strength.

\section{Conclusions}

In this work, we have presented an objective quantification of chaos that shows up in models of the local phase space. This work needs to be buttressed in the future with more sophisticated models that span all six phase space dimensions and account for the Galactic halo as well. This estimation and classification of orbits into strongly chaotic, weakly chaotic and regular, allows us to understand the local phase space in more detail than has been possible before. We implement this technique on models of the solar neighbourhood to conclude that all models that include the spiral pattern exhibit chaoticity and this nature of the local phase space is advanced as an important contributor to the formation of the observed phase space structure. We advance this technique as a blueprint for evaluating the degree of chaos present in kinematic samples that would be collated in the near future by GAIA. 
Acknowledgements. DC is supported by a Royal Society Dorothy Hodgkin Fellowship. IVS is supported by the Tomalla Foundation.

\section{References}

Bissantz, N., Englmaier, P., \& Gerhard, O. 2003, MNRAS, 340, 949

Chakrabarty D. 2004, MNRAS, 352, 427

Chakrabarty, D. 2007, A\&A, 467, 145

Contopoulos G. 2002, Order and Chaos in Dynamical Astronomy (Berlin: Springer-Verlag)

Dehnen, W. 1998, AJ, 115, 2384

Dehnen, W. 2000, AJ, 119, 800

De Simone, R., Wu, X., \& Tremaine, S. 2004, MNRAS, 350, 627

Englmaier, P., \& Gerhard, O. 1999, MNRAS, 304, 512
Evans, N. W., \& Read, J. C. A. 1998, MNRAS, 300, 83

Famaey, B., Jorissen, A., Luri, X., et al. 2005, A\&A, 430, 165

Fux, R. 2001, AJ, 373, 511

Johnston, S., Koribalski, B., Weisberg, J. M., \& Wilson, W. 2001, MNRAS, 322, 715

Lynden-Bell, D., \& Kalnajs, A., 1972, MNRAS, 157, 1

Melnik, A. 2006, Astron. Lett., 32, 7

Quillen, A. C. 2003, AJ, 125, 785

Sellwood, J. A., \& Binney, J. J. 2002, MNRAS, 336, 785

Shirts, R. B. \& Reinhardt, W. P. 1982, J. Chem. Phys., 77, 5204

Sideris, I. V. 2006, Phys. Rev. E, 73, 066217

Sideris, I. V. 2008, Phys. Rev. E, submitted

Skuljan, J., Hearnshaw, J. B., \& Cottrell, P. L. 1999, MNRAS, 308, 731

Vallée, J. P. 2002, ApJ, 566, 261 